\title{
End Graph Effects on Chromatic Polynomials for Strip Graphs of Lattices and their Asymptotic Limits
}

\author{
Shan-Ho Tsai ${ }^{1}$ \\ Institute for Theoretical Physics \\ State University of New York \\ Stony Brook, N. Y. 11794-3840
}

\begin{abstract}
We report exact calculations of the ground state degeneracy per site (exponent of the ground state entropy) $W(\{G\}, q)$ of the $q$-state Potts antiferromagnet on infinitely long strips with specified end graphs for free boundary conditions in the longitudinal direction and free and periodic boundary conditions in the transverse direction. This is equivalent to calculating the chromatic polynomials and their asymptotic limits for these graphs. Making the generalization from $q \in \mathbb{Z}_{+}$to $q \in \mathbb{C}$, we determine the full locus $\mathcal{B}$ on which $W$ is nonanalytic in the complex $q$ plane. We report the first example for this class of strip graphs in which $\mathcal{B}$ encloses regions even for planar end graphs. The bulk of the specific strip graph that exhibits this property is a part of the $\left(3^{3} \cdot 4^{2}\right)$ Archimedean lattice.
\end{abstract}

1 email: tsai@insti.physics.sunysb.edu; address as of May, 1998: Center for Simulational Physics, Department of Physics and Astronomy, University of Georgia, Athens, GA 30602-2451 


\section{INTRODUCTION}

This paper is a continuation of our earlier Ref. [1] and a companion paper to Ref. [2]

on studies of the ground state degeneracy per site, $W(\{G\}, q)$ (exponent of the ground state entropy $S_{0}=k_{B} \ln W$ ) for the $q$-state Potts antiferromagnet (PAF) [3, [1] on infinitely long strip graphs with specified end subgraphs, free boundary conditions in the longitudinal direction, and free and periodic boundary conditions in the transverse direction. The Potts antiferromagnet is of interest for these studies because it is a simple model that exhibits nonzero ground state entropy on a given lattice for sufficiently large values of $q$. The zerotemperature partition function of the $q$-state Potts AF on a graph $G$ is equivalently written as $Z(G, q, T=0)_{P A F}=P(G, q)$, where $P(G, q)$ is the chromatic polynomial [5], giving the number of ways of coloring the $n$-vertex graph $G$ with $q$ colors such that no adjacent vertices have the same color. Hence, the ground state degeneracy per site is $W(\{G\}, q)=$ $\lim _{n \rightarrow \infty} P(G, q)^{1 / n}$ where $\{G\}$ denotes the $n \rightarrow \infty$ limit of the family of graphs $G$ (e.g., a regular lattice, $\{G\}=\Lambda$ with some specified boundary conditions). As before, we shall consider the generalization of $q$ from a postive integer to a complex quantity. In general, $W(\{G\}, q)$ is an analytic function in the $q$ plane except along a certain continuous locus of points, which we denote $\mathcal{B}$.

In Ref. [1] we presented exact calculations of chromatic polynomials and the asymptotic limiting $W$ functions for strip graphs of a variety of regular lattices (with free boundary conditions). For this purpose we developed and applied a generating function method. Specifically, we considered strip graphs of regular lattices of type $s$ of the form

$$
\left(G_{s}\right)_{m ; I}=\left(\prod_{\ell=1}^{m} H\right) I
$$

where $s$ stands for square, triangular, honeycomb, etc. Thus, such a graph is composed of $m$ repetitions of a subgraph $H$ attached to an initial subgraph $I$ on one end, which, by convention, we take to be the right end. In the case of homogeneous strips, $I=H$. As 
is implicit in the above notation, we picture such strip graphs as having a length of $L_{x}$ vertices in the horizontal $(x)$ direction and a width of $L_{y}$ vertices in the transverse (vertical, y) direction. Some further related work is in Ref. [6].

In Ref. [2] we generalized the study in Ref. [1] by considering strip graphs of regular lattices in which there may be special subgraphs on both the left and right ends of the strip:

$$
\left(G_{s}\right)_{m, J, I}=J\left(\prod_{\ell=1}^{m} H\right) I
$$

The end subgraphs $I$ and $J$ may be different from each other and from the repeated subgraph $H$. Furthermore, while the boundary conditions in the longitudinal (horizontal) direction were still free, we studied both free and periodic boundary conditions in the transverse (vertical) direction. We found that while strips of the form (1.1) yield loci $\mathcal{B}$ that consist of arcs and possible line segments which do not depend on the end graph $I$ and which do not enclose regions, strips of the form $(1.2)$ yield loci $\mathcal{B}$ that do, in general, depend on the end graphs $I$ and $J$ and in certain cases do enclose regions. However, for the strips and end graphs considered in Ref. [2], $\mathcal{B}$ only enclosed regions for end graphs that were nonplanar and hence were fundamentally different from the bulk (interior) of the strips, which was planar for both free and periodic boundary conditions in the transverse direction. In the present paper we shall report on further work on this subject and, in particular, our finding of the first case, for this class of strip graphs, in which $\mathcal{B}$ encloses regions for end graphs that are planar, like the rest of the strip. As we shall show, this occurs for strips of a specific type of Archimedean lattice, commonly denoted in the mathematical literature by the symbol $\left(3^{3} \cdot 4^{2}\right)$. Here we recall the definition of an Archimedean lattice as a uniform tiling of the plane by one or more regular polygons, such that each vertex is equivalent to every other vertex [7]. Thus an Archimedean lattice $\Lambda$ is defined by the ordered sequence of polygons that one traverses in making a complete circuit around a vertex in a given (say counterclockwise) direction: 


$$
\Lambda=\left(\prod_{i} p_{i}^{a_{i}}\right)
$$

where in the above circuit, the notation $p_{i}^{a_{i}}$ indicates that the regular $p$-sided polygon $p_{i}$ occurs contiguously $a_{i}$ times (it can also occur noncontiguously). In this paper we shall concentrate on a particular heteropolygonal Archimedean lattice, symbolized in the notation of eq. (1.3) as

$$
\Lambda_{33344}=\left(3^{3} \cdot 4^{2}\right)
$$

An illustration of strips of this lattice is shown in Fig. 1. Evidently, this lattice has coordination number $\Delta=5$, girth $g=3$ (where the girth is the length of minimum-length nontrivial closed circuit on the lattice) and chromatic number $\chi=3$. (The chromatic number $\chi(G)$ of a graph $G$ is defined as the minimum number of colors, $q \in \mathbb{Z}_{+}$which are necessary to color the graph such that no two adjacent vertices have the same color, i.e., the minimum $q \in \mathbb{Z}_{+}$ such that $P(G, q)>0$.) We have obtained rigorous bounds on the ground state entropy of the Potts antiferromagnet on this lattice [8].

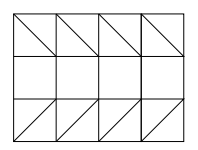

(a)

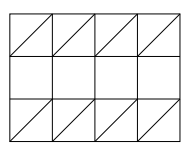

(b)

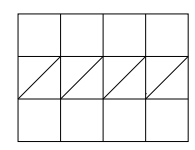

(c)

FIG. 1. Illustrations of strips of the $\left(3^{3} \cdot 4^{2}\right)$ lattice of width $L_{y}=4$. We consider the three types of strips shown in (a), (b) and (c), which we shall refer to as strip graphs of type (33344a), (33344b) and (33344c), respectively. These illustrations show strips with $L_{x}=m+2=5$, corresponding to $n=20$ vertices.

For some relevant definitions and notation, the reader is referred to Refs. [8] and [9]- [11]. In particular, we define the maximal finite real point where $W$ is nonanalytic as $q_{c}$. It will also be convenient to define the function

$$
D_{k}(q)=\sum_{s=0}^{k-2}(-1)^{s}\left(\begin{array}{c}
k-1 \\
s
\end{array}\right) q^{k-2-s}
$$


Some further details are given in Ref. [12]. This paper is organized as follows. In Section II we introduce our notation and briefly summarize the method of generating functions used here. In Section III we present generating functions and the analytic structure of the associated $W$ functions for strips of the $\left(3^{3} \cdot 4^{2}\right)$ lattice with free boundary conditions in the longitudinal and transverse directions of the strips. Section IV reports some results for strips of the $\left(3^{3} \cdot 4^{2}\right)$ lattice with free boundary conditions in the longitudinal direction and periodic boundary conditions in the transverse direction of the strips. Section V contains some conclusions.

\section{GENERATING FUNCTION METHOD AND END SUBGRAPHS}

We denote the chromatic polynomial describing the coloring of the strip graph $\left(G_{s}\right)_{m, J, I}$ with $q$ colors as $P\left(\left(G_{s}\right)_{m, J, I}, q\right)$. In the generating function method, this chromatic polynomial is given by means of an expansion, in terms of an auxiliary variable $x$, of a generating function $\Gamma\left(G_{s, J, I}, q, x\right)$ :

$$
\Gamma\left(G_{s, J, I}, q, x\right)=\sum_{m=0}^{\infty} P\left(\left(G_{s}\right)_{m, J, I}, q\right) x^{m}
$$

where $\Gamma\left(G_{s, J, I}, q, x\right)$ is a rational function of the form

$$
\Gamma\left(G_{s, J, I}, q, x\right)=\frac{\mathcal{N}\left(G_{s, J, I}, q, x\right)}{\mathcal{D}\left(G_{s, J, I}, q, x\right)}
$$

with (suppressing $J, I$ dependence in the notation)

$$
\mathcal{N}\left(G_{s}, q, x\right)=\sum_{j=0}^{j_{\max }} a_{G_{s}, j}(q) x^{j}
$$

and 


$$
\mathcal{D}\left(G_{s}, q, x\right)=1+\sum_{k=1}^{k_{\max }} b_{G_{s}, k}(q) x^{k}
$$

Our calculational methods are described in Refs. [1,2]. Equivalently to eq. (2.1), we calculate the chromatic polynomial as

$$
P\left(\left(G_{s, J, I}\right)_{m}, q\right)=\mathbf{v}_{\mathbf{b}}^{T}(M D)^{m} M \mathbf{v}_{\mathbf{a}}
$$

where $D$ and $M$ are $L \times L$ matrices, with $L$ depending on the width of the strip. Here, as in Ref. [2], we shall restrict ourselves to the cases where $L=5$ for strips with free boundary conditions in the longitudinal and tranverse directions and to the cases where $L=4$ for strips with free boundary condition in the longitudinal direction and periodic boundary condition in the transverse direction, since these are sufficient to exhibit the new phenomenon that we wish to discuss. For the case $L=5$, the matrix $D$ is given by

$$
D=\left(\begin{array}{ccccc}
\frac{1}{q(q-1)(q-2)(q-3)} & 0 & 0 & 0 & 0 \\
0 & \frac{1}{q(q-1)(q-2)} & 0 & 0 & 0 \\
0 & 0 & \frac{1}{q(q-1)(q-2)} & 0 & 0 \\
0 & 0 & 0 & \frac{1}{q(q-1)} & 0 \\
0 & 0 & 0 & 0 & \frac{1}{q(q-1)(q-2)}
\end{array}\right)
$$

whereas for the case $L=4$ the matrix $D$ is the upper left-hand $4 \times 4$ submatrix of $D$ given above in eq. (2.6). The matrix $M$ depends on the specific strip. Factorizing the denominator of the generating function (2.4), we have

$$
\mathcal{D}\left(G_{s, J, I}, q, x\right)=\prod_{r}\left(1-\lambda_{r}(q) x\right)
$$

where the $\lambda_{r}(q)^{\prime} s$ are eigenvalues of the product of matrices $M D$. The subgraphs on the two ends of the strip (defined by $\mathbf{v}_{\mathbf{a}}$ and $\mathbf{v}_{\mathbf{b}}$ ) determine which eigenvalues enter in the product in equation (2.7). Using this method, we have calculated chromatic polynomials and their asymptotic limits for various strip graphs of the form $\left(G_{s}^{\prime}\right)_{m} \equiv\left(G_{s, J, I}\right)_{m}=J\left(\prod_{\ell=1}^{m} H\right) I$, with 
$\left(G_{s}^{\prime}\right)_{0} \equiv J I$, where the end graphs $I$ and $J$ are, in general, different. We follow the same notation as in Ref. [2] for the vectors that describe the subgraphs on the ends of the strips: (i) for the case $L=5, \mathbf{v}_{a}$ and $\mathbf{v}_{b}$ will be chosen from the following vectors:

$$
\begin{aligned}
& \mathbf{v}_{\mathbf{1}}=(1,0,0,0,0)^{T} \\
& \mathbf{v}_{\mathbf{2}}=(0,1,0,0,0)^{T} \\
& \mathbf{v}_{\mathbf{3}}=(0,0,1,0,0)^{T} \\
& \mathbf{v}_{\mathbf{4}}=(0,0,0,1,0)^{T} \\
& \mathbf{v}_{\mathbf{5}}=(0,0,0,0,1)^{T} \\
& \mathbf{v}_{\mathbf{6}}=(1,1,0,0,0)^{T} \\
& \mathbf{v}_{\mathbf{7}}=(1,0,1,0,0)^{T} \\
& \mathbf{v}=(1,1,1,1,1)^{T}
\end{aligned}
$$

(ii) for the case $L=4, \mathbf{v}_{a}$ and $\mathbf{v}_{b}$ will be chosen from the following vectors:

$$
\begin{aligned}
& \mathbf{v}_{\mathbf{1}}=(1,0,0,0)^{T} \\
& \mathbf{v}_{\mathbf{2}}=(0,1,0,0)^{T} \\
& \mathbf{v}_{\mathbf{3}}=(0,0,1,0)^{T} \\
& \mathbf{v}_{\mathbf{4}}=(0,0,0,1)^{T}
\end{aligned}
$$




$$
\begin{aligned}
& \mathbf{v}_{\mathbf{5}}=(1,1,0,0,)^{T} \\
& \mathbf{v}_{\mathbf{6}}=(1,0,1,0)^{T} \\
& \mathbf{v}=(1,1,1,1)^{T}
\end{aligned}
$$

For $\mathbf{v}$, the end subgraph is the same as the transverse set of edges and vertices. The end graphs corresponding to the vectors in eqs. (2.8)-(2.15) were illustrated in Ref. [2].

For strip graphs with free boundary conditions in the $x$ and $y$ directions $\left(\mathrm{FBC}_{x}\right.$ and $\mathrm{FBC}_{y}$ ), we shall restrict ourselves to cases where the product of matrices $M D$ is a $5 \times 5$ matrix. For strip graphs with free boundary conditions in the $x$ direction $\left(\mathrm{FBC}_{x}\right)$ and periodic boundary conditions in the $y$ direction $\left(\mathrm{PBC}_{y}\right)$ we shall restrict ourselves to cases where the product of matrices $M D$ are $4 \times 4$ matrices. With these preliminaries covered, we proceed to our new results.

\section{STRIPS OF THE $\left(3^{3} \cdot 4^{2}\right)$ LATTICE OF WIDTH $L_{y}=4$ AND FBC FB $_{y}$}

We consider three types of strips of the $\left(3^{3} \cdot 4^{2}\right)$ lattice of width $L_{y}=4$. Sections of these strips are illustrated in Figs. 1(a), 1(b) and 1(c) and will be referred to as strip graphs of type $(33344 a),(33344 b)$ and $(33344 c)$, respectively.

\section{A. Type $(33344 a)$}

For a strip graph of type $(33344 a)$, width $L_{y}=4$ and end subgraphs described by vectors v, i.e., end subgraphs as shown in Fig. 1(a), we obtain a generating function of the form of Eqs. (2.2), (2.3) and (2.4), with $j_{\max }=2, k_{\max }=3$ and coefficients

$$
a_{33344 a(4), 0}=q(q-1)\left(q^{2}-3 q+3\right)(q-2)^{4}
$$




$$
\begin{gathered}
a_{33344 a(4), 1}=-q(q-1)\left(2 q^{5}-21 q^{4}+86 q^{3}-171 q^{2}+164 q-59\right)(q-2)^{3}, \\
a_{33344 a(4), 2}=q(q-3)\left(q^{2}-5 q+7\right)(q-1)^{3}(q-2)^{5}, \\
b_{33344 a(4), 1}=-q^{4}+9 q^{3}-34 q^{2}+66 q-55, \\
b_{33344 a(4), 2}=(q-2)(q-3)\left(2 q^{2}-11 q+16\right)\left(q^{2}-4 q+5\right), \\
b_{33344 a(4), 3}=-(q-3)\left(q^{2}-5 q+7\right)(q-2)^{5} .
\end{gathered}
$$

Three of the five eigenvalues of $M D$ are the inverses of the three roots of $1+b_{33344 a(4), 1} x+$ $b_{33344 a(4), 2} x^{2}+b_{33344 a(4), 3} x^{3}$. These eigenvalues contribute to the generating functions for all end subgraphs considered here. The other two eigenvalues are $\lambda_{4}=1$ and $\lambda_{5}=(q-2)^{2}$, which only contribute to the generating functions for some end subgraphs. In Table \& we show for some illustrative planar and non-planar end subgraphs, whether or not $\lambda_{4}$ and $\lambda_{5}$ contribute to the generating function and some features of the locus $\mathcal{B}$ for each case. 
TABLE I. Illustrative end subgraphs for a strip of the $\left(3^{3} \cdot 4^{2}\right)$ lattice of type (33344a) and width $L_{y}=4$. The notation $\mathbf{v}_{\mathbf{J}}, \mathbf{v}_{\mathbf{I}}$ means that the vectors $\mathbf{v}_{J}$ and $\mathbf{v}_{I}$ describe the subgraphs on the left- and right-hand end of the strip. P stands for planar and NP for non-planar. The third and fourth columns show respectively whether or not $\lambda_{4}$ and $\lambda_{5}$ enter in the generating function. The fifth column lists some features of $\mathcal{B}$.

\begin{tabular}{|c|c|c|c|c|}
\hline$J, I$ & planarity & does $\lambda_{4}$ enter? & does $\lambda_{5}$ enter? & features of $\mathcal{B}$ \\
\hline $\mathbf{v}, \mathbf{v}$ & $\mathrm{P}, \mathrm{P}$ & $\mathrm{N}$ & $\mathrm{N}$ & $\operatorname{arcs}$ \\
\hline $\mathbf{v}, \mathbf{v}_{\mathbf{i}}, i=1,2,3,4$ & $\mathrm{P}, \mathrm{NP}$ & $\mathrm{N}$ & $\mathrm{N}$ & $\operatorname{arcs}$ \\
\hline $\mathbf{v}, \mathbf{v}_{\mathbf{i}}, i=5,6,7$ & $\mathrm{P}, \mathrm{P}$ & $\mathrm{N}$ & $\mathrm{N}$ & $\operatorname{arcs}$ \\
\hline $\mathbf{v}_{\mathbf{1}}, \mathbf{v}_{\mathbf{i}}, i=1,2,3,4$ & $\mathrm{NP}, \mathrm{NP}$ & $\mathrm{Y}$ & $\mathrm{N}$ & arcs and one enclosed region \\
\hline $\mathbf{v}_{\mathbf{1}}, \mathbf{v}_{\mathbf{i}}, i=5,6,7$ & $\mathrm{NP}, \mathrm{P}$ & $\mathrm{N}$ & $\mathrm{N}$ & $\operatorname{arcs}$ \\
\hline $\mathbf{v}_{\mathbf{2}}, \mathbf{v}_{\mathbf{i}}, i=2,3$ & $\mathrm{NP}, \mathrm{NP}$ & $\mathrm{Y}$ & $\mathrm{Y}$ & arcs and three enclosed regions \\
\hline $\mathbf{v}_{\mathbf{2}}, \mathbf{v}_{\mathbf{i}}, i=6,7$ & $\mathrm{NP}, \mathrm{P}$ & $\mathrm{N}$ & $\mathrm{Y}$ & arcs and two enclosed regions \\
\hline $\mathbf{v}_{3}, v_{3}$ & $\mathrm{NP}, \mathrm{NP}$ & $\mathrm{Y}$ & $\mathrm{Y}$ & arcs and three enclosed regions \\
\hline $\mathbf{v}_{\mathbf{4}}, \mathbf{v}_{\mathbf{i}}, i=2,3,4$ & $\mathrm{NP}, \mathrm{NP}$ & $\mathrm{Y}$ & $\mathrm{N}$ & arcs and one enclosed region \\
\hline $\mathbf{v}_{\mathbf{4}}, \mathbf{v}_{\mathbf{i}}, i=6,7$ & $\mathrm{NP}, \mathrm{P}$ & $\mathrm{N}$ & $\mathrm{N}$ & $\operatorname{arcs}$ \\
\hline $\mathbf{v}_{\mathbf{5}}, \mathbf{v}_{\mathbf{i}}, i=2,3,4$ & $\mathrm{P}, \mathrm{NP}$ & $\mathrm{N}$ & $\mathrm{N}$ & $\operatorname{arcs}$ \\
\hline $\mathbf{v}_{\mathbf{5}}, \mathbf{v}_{\mathbf{i}}, i=5,6,7$ & $\mathrm{P}, \mathrm{P}$ & $\mathrm{N}$ & $\mathrm{N}$ & $\operatorname{arcs}$ \\
\hline $\mathbf{v}_{\mathbf{6}}, \mathbf{v}_{\mathbf{i}}, i=2,3$ & $\mathrm{P}, \mathrm{NP}$ & $\mathrm{N}$ & $\mathrm{Y}$ & arcs and two enclosed regions \\
\hline $\mathbf{v}_{\mathbf{6}}, \mathbf{v}_{\mathbf{i}}, i=6,7$ & $\mathrm{P}, \mathrm{P}$ & $\mathrm{N}$ & $\mathrm{Y}$ & arcs and two enclosed regions \\
\hline $\mathbf{v}_{\mathbf{7}}, \mathbf{v}_{\mathbf{i}}, i=2,3$ & $\mathrm{P}, \mathrm{NP}$ & $\mathrm{N}$ & $\mathrm{Y}$ & arcs and two enclosed regions \\
\hline $\mathrm{v}_{7}, \mathrm{v}_{7}$ & $\mathrm{P}, \mathrm{P}$ & $\mathrm{N}$ & $\mathrm{Y}$ & arcs and two enclosed regions \\
\hline
\end{tabular}




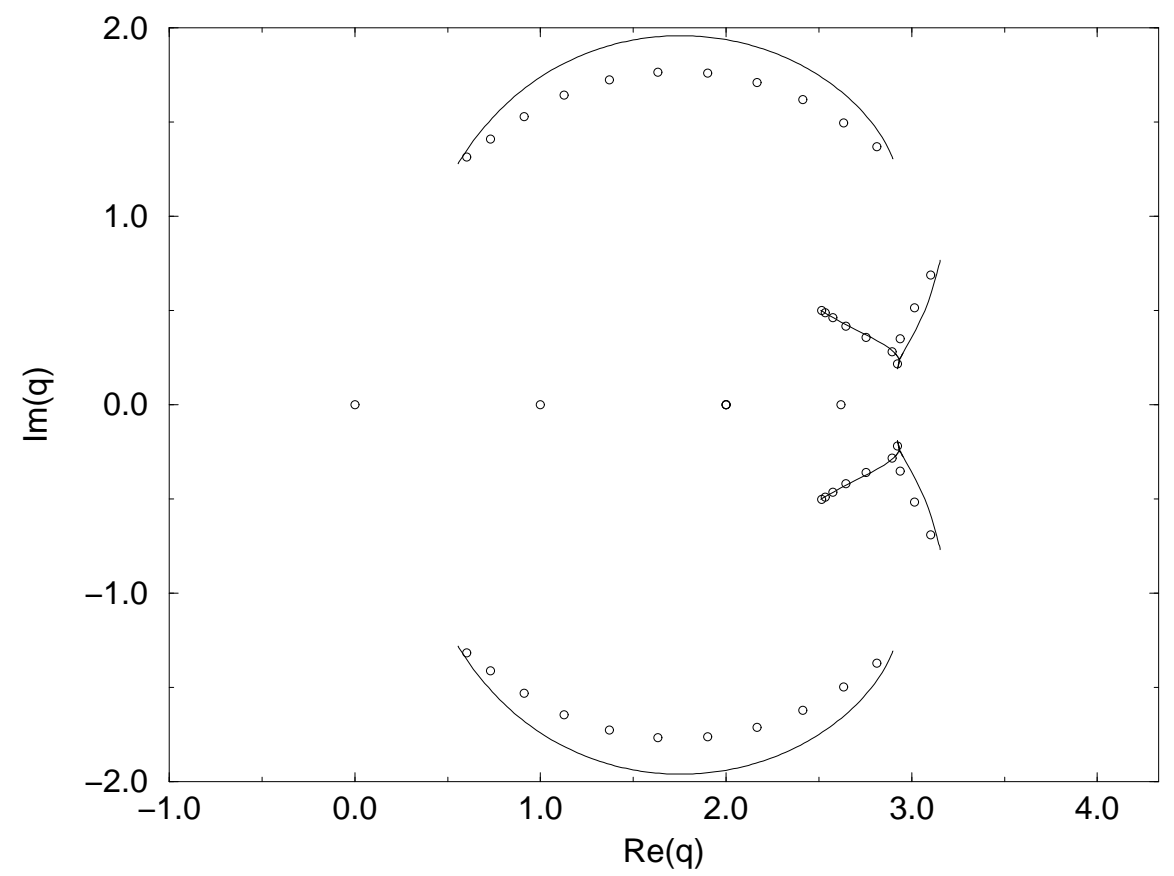

FIG. 2. Analytic structure of the function $W\left(\left\{G_{33344 a(4)}^{\prime}\right\}, q\right)$ for the strip of the $\left(3^{3} \cdot 4^{2}\right)$ lattice of type (33344a), width $L_{y}=4$ and length $L_{x}=\infty$. End subgraphs are such that $\lambda_{4}$ and $\lambda_{5}$ do not appear in the generating function. For comparison, the zeros of the chromatic polynomial for a strip with $m=10$ repeating units ( $n=48$ vertices) and subgraphs described by $\mathbf{v}$ on the right and left ends of the strip, are shown. 


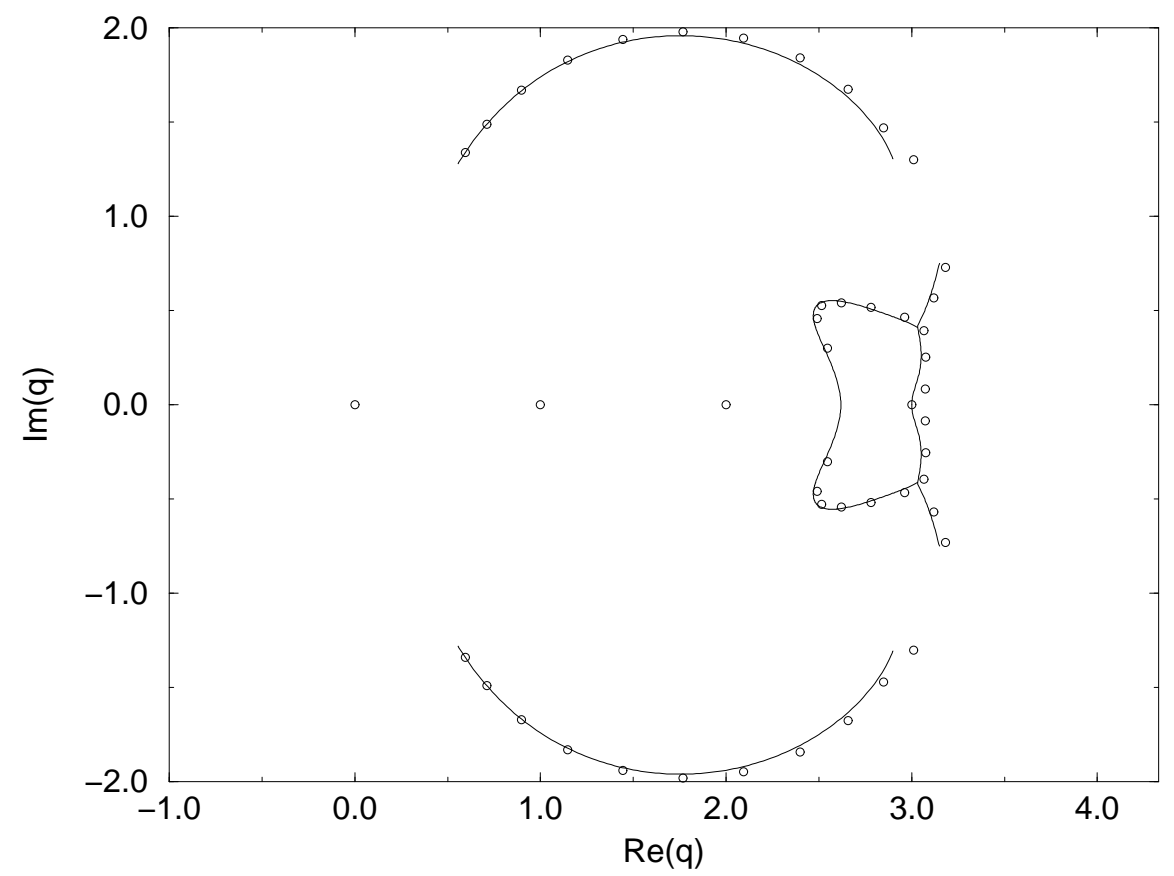

FIG. 3. Analytic structure of the function $W\left(\left\{G_{33344 a(4)}^{\prime}\right\}, q\right)$ for the strip of the $\left(3^{3} \cdot 4^{2}\right)$ lattice of type (33344a), width $L_{y}=4$ and length $L_{x}=\infty$. End subgraphs are such that $\lambda_{4}$ appears in the generating function, but $\lambda_{5}$ does not. For comparison, the zeros of the chromatic polynomial for a strip with $m=10$ repeating units ( $n=48$ vertices) and subgraphs described by $\mathbf{v}_{\mathbf{1}}$ on the right and left ends of the strip, are shown. 




FIG. 4. Analytic structure of the function $W\left(\left\{G_{33344 a(4)}^{\prime}\right\}, q\right)$ for the strip of the $\left(3^{3} \cdot 4^{2}\right)$ lattice of type (33344a), width $L_{y}=4$ and length $L_{x}=\infty$. End subgraphs are such that $\lambda_{5}$ appears in the generating function, but $\lambda_{4}$ does not. For comparison, the zeros of the chromatic polynomial for a strip with $m=10$ repeating units ( $n=48$ vertices) and subgraphs described by $\mathbf{v}_{\mathbf{6}}$ on the right and left ends of the strip, are shown. 


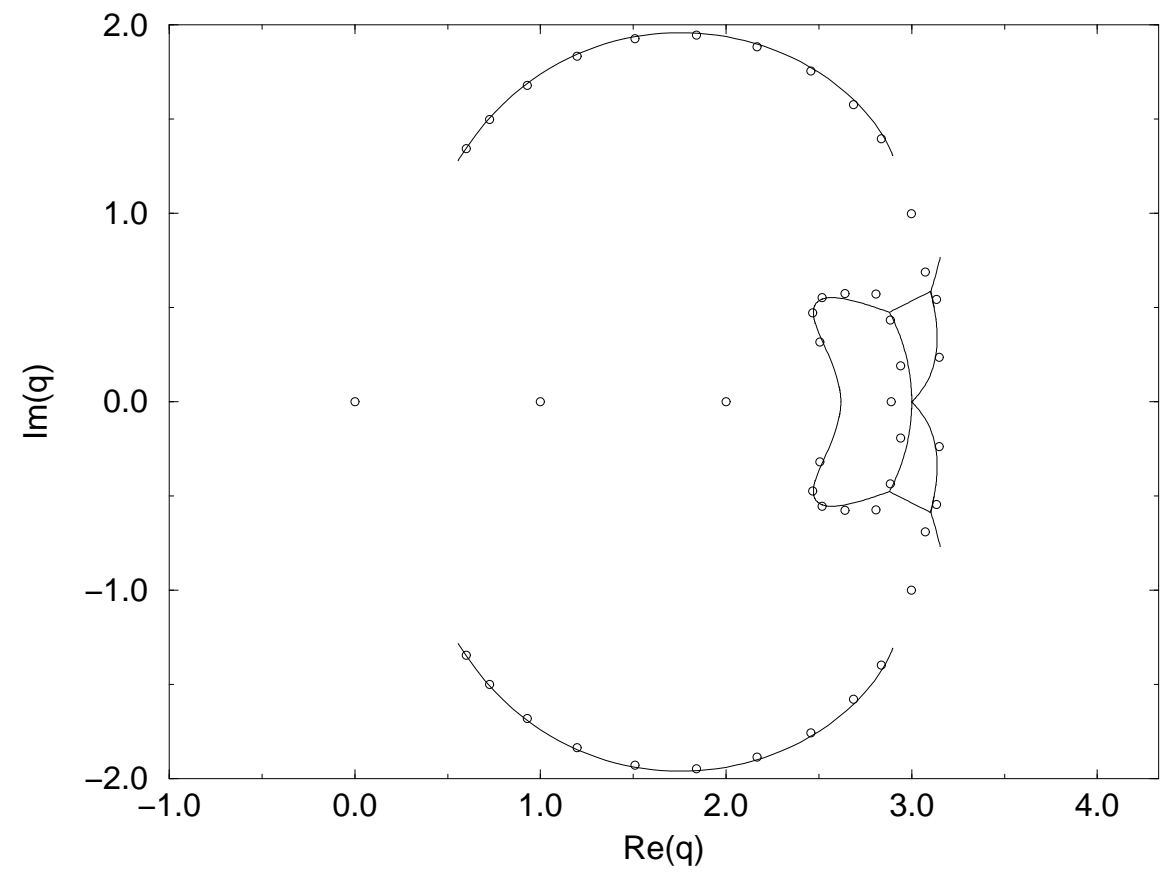

FIG. 5. Analytic structure of the function $W\left(\left\{G_{33344 a(4)}^{\prime}\right\}, q\right)$ for the strip of the $\left(3^{3} \cdot 4^{2}\right)$ lattice of type (33344a), width $L_{y}=4$ and length $L_{x}=\infty$. End subgraphs are such that $\lambda_{4}$ and $\lambda_{5}$ appear in the generating function. For comparison, the zeros of the chromatic polynomial for a strip with $m=10$ repeating units ( $n=46$ vertices) and subgraphs described by $\mathbf{v}_{\mathbf{2}}$ on the right and left ends of the strip, are shown.

Figs. 2, 3, 1 and 5 show the analytic structure of $W\left(\left\{G_{33344 a(4)}^{\prime}\right\}, q\right)$ for a strip of the $\left(3^{3} \cdot 4^{2}\right)$ lattice of type $(33344 a)$ and width $L_{y}=4$. Fig. 2 corresponds to end subgraphs that do not yield $\lambda_{4}$ and $\lambda_{5}$ in the generating function. In these cases the locus $\mathcal{B}$ is formed by pairs of complex conjugate arcs that do not separate the $q$ plane into separate regions. One can observe also the features that (i) there are two complex-conjugate multiple points 13. on $\mathcal{B}$ at $q_{m} \simeq 2.93 \pm 0.24 i$ and (ii) $\mathcal{B}$ does not cross the real $q$ axis. These multiple points have the same topology as the one shown in Fig. 7(b). Fig. 3 corresponds to end 
subgraphs that yield $\lambda_{4}$ but do not yield $\lambda_{5}$ in the generating function. In these cases the locus $\mathcal{B}$ is comprised by arcs which enclose one self-conjugate region, where $\lambda_{4}$ is the leading eigenvalue. The locus $\mathcal{B}$ crosses the real $q$ axis at $q=\frac{3+\sqrt{5}}{2}=2.618 \ldots$ and at $q=3$. Thus $q_{c}=3$ for end subgraphs that yield $\mathcal{B}$ shown in this figure. Fig. 4 corresponds to end subgraphs that yield $\lambda_{5}$ but do not yield $\lambda_{4}$ in the generating function. In these cases the locus $\mathcal{B}$ is comprised by arcs which enclose one pair of complex-conjugate regions, where $\lambda_{5}$ is the leading eigenvalue. The locus $\mathcal{B}$ crosses the real $q$ axis at one point, namely at $q=3$. When the end subgraphs are such that both $\lambda_{4}$ and $\lambda_{5}$ appear in the generating function, the boundary $\mathcal{B}$ is comprised by arcs which enclose one self-conjugate region and one pair of complex-conjugate regions, as shown in Fig. 5. In the self-conjugate region the leading eigenvalue is $\lambda_{4}$, whereas in the complex-conjugate regions $\lambda_{5}$ is leading. The locus $\mathcal{B}$ crosses the real $q$ axis at $q=\frac{3+\sqrt{5}}{2}=2.618 \ldots$ and at $q=3$. It is interesting to note that in all cases where $\mathcal{B}$ crosses the real $q$ axis, the rightmost crossing point is at $q_{c}=3$.

The locus $\mathcal{B}$ shown in Fig. 2 contains arc endpoints at $q_{e 1} \simeq 0.556+1.281 i, q_{e 2} \simeq$ $2.898+1.307 i, q_{e 3} \simeq 3.154+0.767 i, q_{e 4} \simeq 2.923+0.190 i, q_{e 5} \simeq 2.509+0.502 i$ and at the complex-conjugates of these points. In the locus $\mathcal{B}$ shown in Fig. 3 the arc endpoints occur at $q_{e 1}, q_{e 2}, q_{e 3}$ and their complex-conjugates. The points $q_{e 4}$ and $q_{e 5}$ (and their complexconjugates) lie within the region where $\lambda_{4}$ is leading and do not correspond to arc endpoints. The locus $\mathcal{B}$ shown in Fig. 7 contains arc endpoints at $q_{e 1}, q_{e 2}, q_{e 3}, q_{e 5}$ and their complexconjugates. The point $q_{e 4}$ and its complex-conjugate lie within the regions where $\lambda_{5}$ is leading and do not correspond to arc endpoints. The locus $\mathcal{B}$ shown in Fig. 5 contains arc endpoints at $q_{e 1}, q_{e 2}, q_{e 3}$, and their complex-conjugates. The points $q_{e 4}$ and $q_{e 5}$ (and their complex-conjugates) lie within the region where $\lambda_{4}$ is leading and do not correspond to arc endpoints.

For a strip of the triangular lattice of width $L_{y}=4$, as discussed in Ref. [2], we only obtained loci $\mathcal{B}$ comprised by arcs separating the complex $q$ plane into disconnected regions 
when subgraphs on the right and left ends of the strip involved non-planar graphs. For a strip of the $\left(3^{3} \cdot 4^{2}\right)$ lattice of type $(33344 a)$ and width $L_{y}=4$, planar subgraphs on one end or on both ends of the strip can yield arcs which enclose regions in $\mathcal{B}$.

\section{B. Type $(33344 b)$}

For a strip graph of type (33344b), width $L_{y}=4$ and end subgraphs described by vectors v, i.e., as shown in Fig. 田(b), we obtain the same generating function as for the strip graph (33344a), with coefficients given in Eqs. (3.1)-(3.6). The five eingenvalues of $M D$ are also the same as for the strip graph of type (33344a) and width $L_{y}=4$. However, end subgraphs that yield $\lambda_{4}$ or $\lambda_{5}$ or both in the generating functions are not identical for these two types of strips. In Table 【 $\llbracket$ we show for some illustrative planar and non-planar end subgraphs, whether or not $\lambda_{4}$ and $\lambda_{5}$ contribute to the generating function and some features of the loci $\mathcal{B}$ for each case.

TABLE II. Illustrative end subgraphs for a strip of the $\left(3^{3} \cdot 4^{2}\right)$ lattice of type $(33344 b)$ and width $L_{y}=4$. The notation is the same as in Table $\mathrm{Q}$.

\begin{tabular}{|ccccc|}
\hline \hline$J, I$ & planarity & does $\lambda_{4}$ enter? & does $\lambda_{5}$ enter? & features of $\mathcal{B}$ \\
\hline \hline $\mathbf{v}, \mathbf{v}$ & $\mathrm{P}, \mathrm{P}$ & $\mathrm{N}$ & $\mathrm{N}$ & arcs \\
$\mathbf{v}_{\mathbf{i}}, \mathbf{v}_{\mathbf{i}}, i=1,4$ & $\mathrm{NP}, \mathrm{NP}$ & $\mathrm{Y}$ & $\mathrm{Y}$ & arcs and three enclosed regions \\
$\mathbf{v}_{\mathbf{i}}, \mathbf{v}_{\mathbf{i}}, i=2,3$ & $\mathrm{NP}, \mathrm{NP}$ & $\mathrm{Y}$ & $\mathrm{N}$ & arcs and one enclosed region \\
$\mathbf{v}_{\mathbf{5}}, \mathbf{v}_{\mathbf{5}}$ & $\mathrm{P}, \mathrm{P}$ & $\mathrm{N}$ & $\mathrm{Y}$ & arcs and two enclosed regions \\
$\mathbf{v}_{\mathbf{i}}, \mathbf{v}_{\mathbf{i}}, i=6,7$ & $\mathrm{P}, \mathrm{P}$ & $\mathrm{N}$ & $\mathrm{N}$ & arcs \\
\hline \hline
\end{tabular}


For strips of the $\left(3^{3} \cdot 4^{2}\right)$ lattice of width $L_{y}=4$ and types $(33344 a)$ and $(33344 b)$ the analytic struture of $W\left(\left\{G_{33344(4)}^{\prime}\right\}, q\right)$ can have enclosed regions when (i) the subgraphs on one end of the strip involve a non-planar graph and on the other end a planar graph, (ii) the subgraphs on both ends involve planar graphs or (iii) the subgraphs on both ends involve non-planar graphs.

\section{Type $(33344 c)$}

For a strip graph of type (33344c) and subgraphs described by vectors v, i.e., as shown in Fig. 1(c), we obtain a generating function of the form of Eqs. (2.2), (2.3) and (2.4), with $j_{\max }=3, k_{\max }=4$ and coefficients

$$
\begin{gathered}
a_{33344 c(4), 0}=q(q-1)(q-2)^{2}\left(q^{2}-3 q+3\right)^{2} \\
a_{33344 c(4), 1}=-q(q-1)(q-2)\left(3 q^{7}-38 q^{6}+208 q^{5}-638 q^{4}+1185 q^{3}-1330 q^{2}+829 q-217\right) \\
a_{33344 c(4), 2}=q(q-1)(3 q-4)\left(q^{2}-3 q+3\right)\left(q^{3}-7 q^{2}+15 q-8\right)(q-2)^{4} \\
a_{33344 c(4), 3}=-q\left(q^{2}-5 q+5\right)^{2}(q-1)^{3}(q-2)^{6} \\
b_{33344 c(4), 1}=-q^{4}+8 q^{3}-29 q^{2}+56 q-46 \\
b_{33344 c(4), 2}=3 q^{6}-38 q^{5}+208 q^{4}-634 q^{3}+1136 q^{2}-1122 q+465 \\
b_{33344 c(4), 3}=-3 q^{8}+52 q^{7}-395 q^{6}+1720 q^{5}-4702 q^{4}+8264 q^{3}-9101 q^{2}+5718 q-1561
\end{gathered}
$$




$$
b_{33344 c(4), 4}=(q-2)^{6}\left(q^{2}-5 q+5\right)^{2}
$$

Four of the five eigenvalues of $M D$ are the inverses of the four roots of $1+b_{33344 c(4), 1} x+$ $b_{33344 c(4), 2} x^{2}+b_{33344 c(4), 3} x^{3}+b_{33344 c(4), 4} x^{4}$. These eigenvalues contribute to the generating functions for all end subgraphs considered here. The other eigenvalue is $\lambda_{5}=1$, which contributes to the generating functions for some end subgraphs. In Table III we show for some illustrative planar and non-planar end subgraphs, whether or not $\lambda_{5}$ contributes to the generating function and some features of the locus $\mathcal{B}$ for each case.

TABLE III. Illustrative end subgraphs for a strip of the $\left(3^{3} \cdot 4^{2}\right)$ lattice of type $(33344 c)$ and width $L_{y}=4$. The notation is the same as in Table 1 .

\begin{tabular}{|cccc|}
\hline \hline \multicolumn{1}{c}{$J, I$} & planarity & does $\lambda_{5}$ enter? & features of $\mathcal{B}$ \\
\hline \hline $\mathbf{v}, \mathbf{v}$ & $\mathrm{P}, \mathrm{P}$ & $\mathrm{N}$ & $\operatorname{arcs}$ \\
$\mathbf{v}_{\mathbf{i}}, \mathbf{v}_{\mathbf{i}}, i=1,2,3,4$ & $\mathrm{NP}, \mathrm{NP}$ & $\mathrm{Y}$ & $\operatorname{arcs}$ and one enclosed region \\
$\mathbf{v}_{\mathbf{i}}, \mathbf{v}_{\mathbf{i}}, i=5,6,7$ & $\mathrm{P}, \mathrm{P}$ & $\mathrm{N}$ & $\operatorname{arcs}$ \\
$\mathbf{v}, \mathbf{v}_{\mathbf{i}}, i=1,2,3,4$ & $\mathrm{P}, \mathrm{NP}$ & $\mathrm{N}$ & $\operatorname{arcs}$ \\
$\mathbf{v}, \mathbf{v}_{\mathbf{i}}, i=5,6,7$ & $\mathrm{P}, \mathrm{P}$ & $\mathrm{N}$ & $\operatorname{arcs}$ \\
$\mathbf{v}_{\mathbf{1}}, \mathbf{v}_{\mathbf{i}}, i=2,3,4$ & $\mathrm{NP}, \mathrm{NP}$ & $\mathrm{Y}$ & $\operatorname{arcs}$ and one enclosed region \\
$\mathbf{v}_{\mathbf{1}}, \mathbf{v}_{\mathbf{i}}, i=5,6,7$ & $\mathrm{NP}, \mathrm{P}$ & $\mathrm{N}$ & $\operatorname{arcs}$ \\
$\mathbf{v}_{\mathbf{2}}, \mathbf{v}_{\mathbf{i}}, i=3,4$ & $\mathrm{NP}, \mathrm{NP}$ & $\mathrm{Y}$ & $\operatorname{arcs}$ and one enclosed region \\
$\mathbf{v}_{\mathbf{2}}, \mathbf{v}_{\mathbf{i}}, i=5,6,7$ & $\mathrm{NP}, \mathrm{P}$ & $\mathrm{N}$ & $\operatorname{arcs}$ \\
$\mathbf{v}_{\mathbf{3}}, \mathbf{v}_{\mathbf{4}}$ & $\mathrm{NP}, \mathrm{NP}$ & $\mathrm{Y}$ & $\operatorname{arcs}$ \\
$\mathbf{v}_{\mathbf{3}}, \mathbf{v}_{\mathbf{i}}, i=5,6,7$ & $\mathrm{NP}, \mathrm{P}$ & $\mathrm{N}$ & $\operatorname{arcs}$ \\
$\mathbf{v}_{\mathbf{4}}, \mathbf{v}_{\mathbf{i}}, i=5,6,7$ & $\mathrm{NP}, \mathrm{P}$ & $\mathrm{N}$ & $\operatorname{arcs}$ \\
$\mathbf{v}_{\mathbf{5}}, \mathbf{v}_{\mathbf{i}}, i=6,7$ & $\mathrm{P}, \mathrm{P}$ & $\mathrm{N}$ & $\mathrm{N}$ \\
$\mathbf{v}_{\mathbf{6}}, \mathbf{v}_{\mathbf{7}}$ & $\mathrm{P}, \mathrm{P}$ & & arcs \\
\hline \hline \hline
\end{tabular}



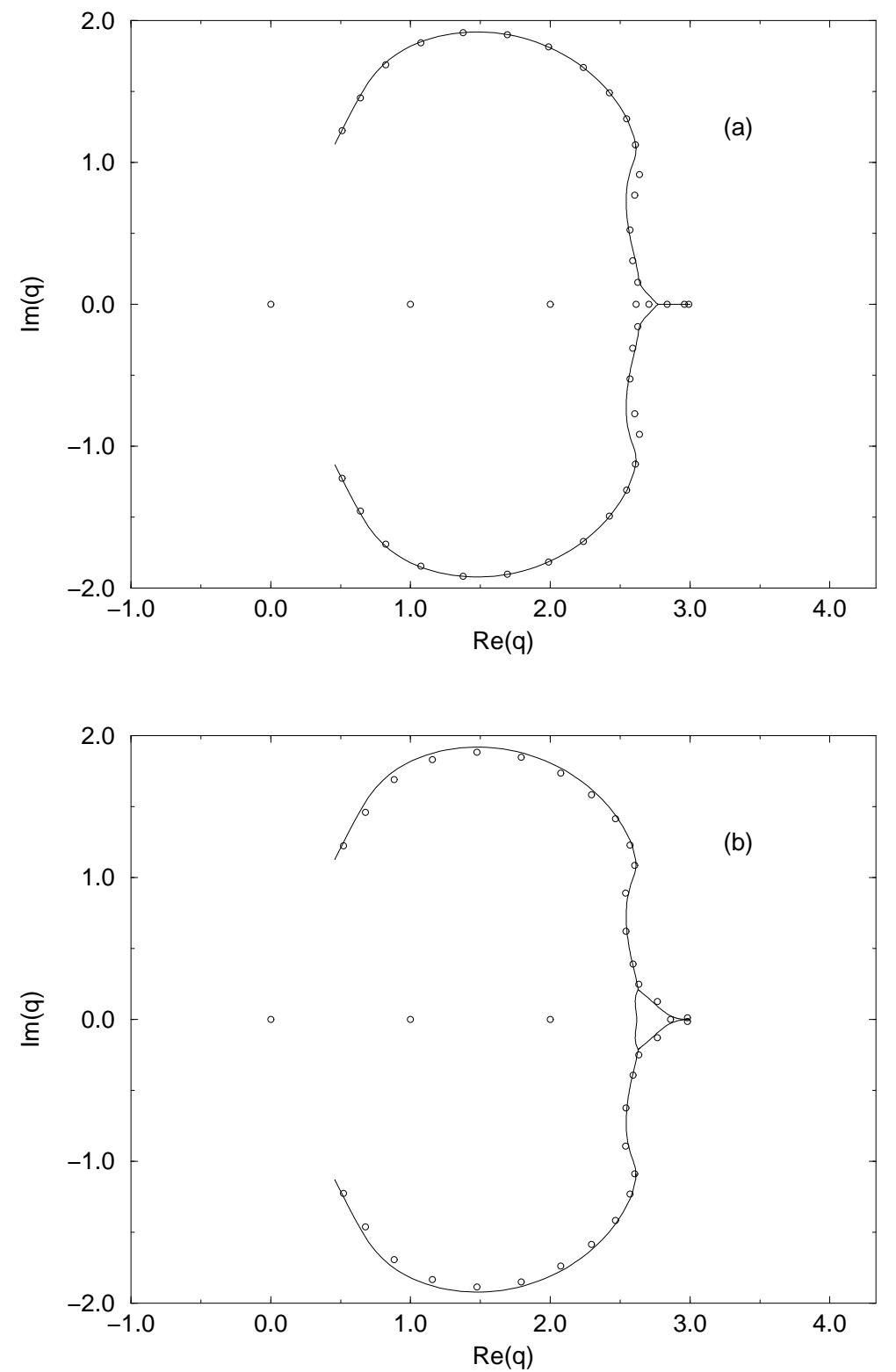

FIG. 6. Analytic structure of the function $W\left(\left\{G_{33344 c(4)}^{\prime}\right\}, q\right)$ for a strip of the $\left(3^{3} \cdot 4^{2}\right)$ lattice of type (33344c), width $L_{y}=4$ and length $L_{x}=\infty$. End subgraphs in (a) [(b)] are such that $\lambda_{5}$ does not [does] appear in the generating function. For comparison, zeros of the chromatic polynomial for a strip with $m=8$ repeating units ( $n=40$ vertices in (a) and $n=36$ vertices in (b)) and end subgraphs described by (a) $\mathbf{v}_{\mathbf{6}}$ $\left[(b) \mathbf{v}_{\mathbf{2}}\right]$ on the right and left ends of the strip, are shown. 

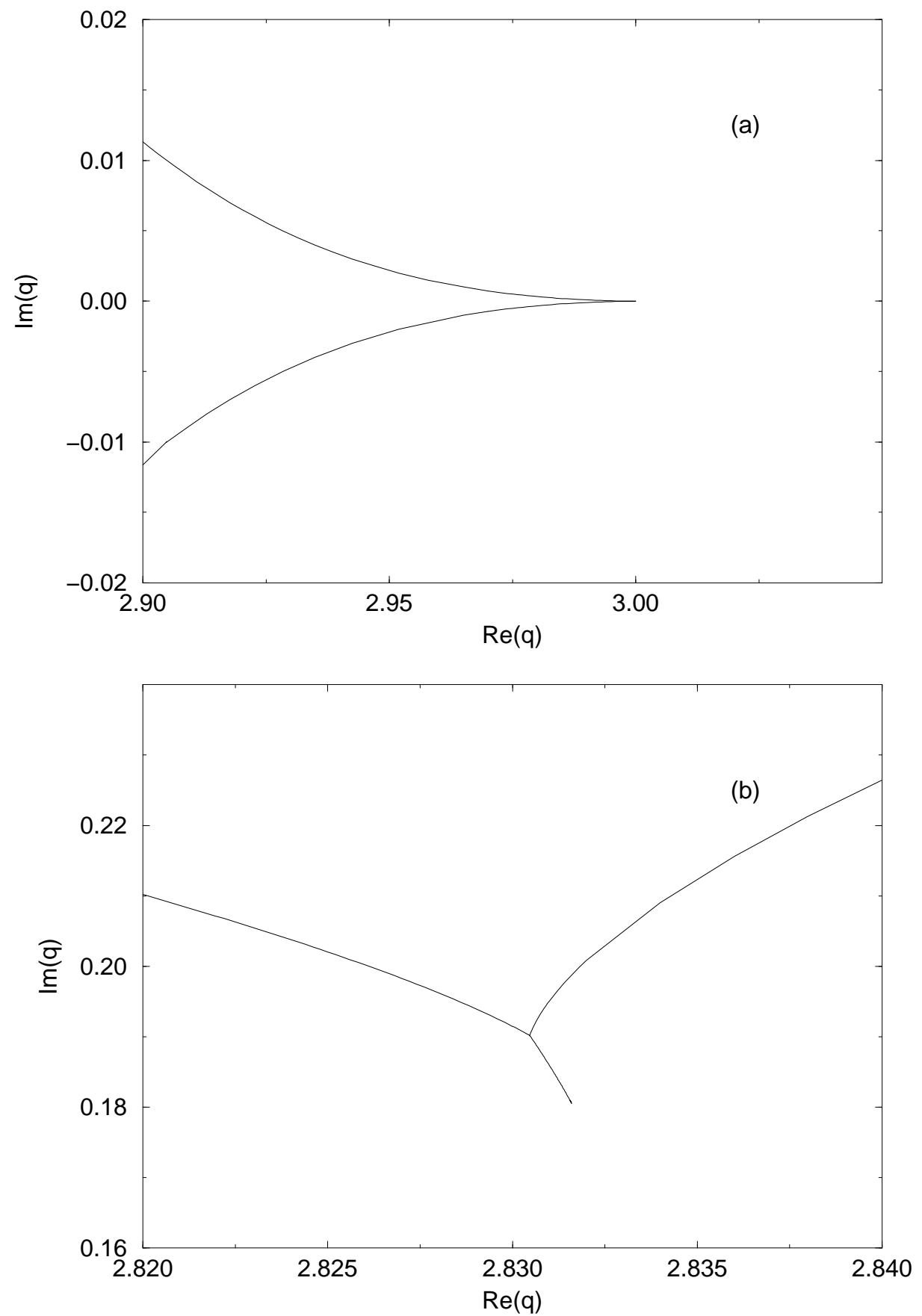

FIG. 7. Enlargement of the region in the complex $q$ plane near the (a) cusp in Fig. 6(b) and (b) multiple point with positive imaginary part in Fig. 8(a). 
Figs. 6(a) and 6(b) show the analytic structure of the $W\left(\left\{G_{33344 c(4)}^{\prime}\right\}, q\right)$ function for a strip of the $\left(3^{3} \cdot 4^{2}\right)$ lattice of type $(33344 c)$ and width $L_{y}=4$. Fig. 6(a) corresponds to end subgraphs that do not yield $\lambda_{5}$ in the generating function. In these cases $\mathcal{B}$ is formed by a self-conjugate arc and a line segment on the real $q$ axis, extending from the multiple point $q \simeq 2.772$ to $q=3$. Fig. 6(b) corresponds to end subgraphs that include $\lambda_{5}$ in the generating function. In these cases $\mathcal{B}$ is comprised of arcs which enclose one self-conjugate

region, where $\lambda_{5}$ is leading. The locus $\mathcal{B}$ crosses the real $q$ axis at $q=\frac{3+\sqrt{5}}{2}=2.618 \ldots$ and at $q=3$. At the latter point the boundary $\mathcal{B}$ forms a cusp, as shown in Fig. W(a). At the point $q=3$ the denominator of the generating function for the cases which yield boundary $\mathcal{B}$ as shown in Fig. 6(a), has the factored form $\mathcal{D}=(x-1)^{4}$. Similarly, for the cases which yield boundary $\mathcal{B}$ as shown in Fig. 6(b) the denominator factors as $\mathcal{D}=(x-1)^{5}$ at $q=3$. For this type of strip graphs all subgraphs on the two ends of the strips yield $q_{c}=\chi=3$. Another example of a boundary $\mathcal{B}$ which includes cusps was discussed in Ref. [14.

\section{STRIP OF THE $\left(3^{3} \cdot 4^{2}\right)$ LATTICE WITH PERIODIC BOUNDARY CONDITIONS IN THE TRANSVERSE DIRECTION}

For a strip of the $\left(3^{3} \cdot 4^{2}\right)$ lattice of type $(33344 b)$ with periodic boundary conditions in the transverse direction, transverse cross sections forming squares, and subgraphs on the two free ends of the strip described by the vector $\mathbf{v}$, we obtain a generating function with $j_{\max }=2, k_{\max }=3$, and coefficients

$$
\begin{gathered}
a_{33344(4), c, 0}=q(q-1)(q-2)\left(q^{5}-11 q^{4}+52 q^{3}-134 q^{2}+189 q-115\right) \\
a_{33344(4), c, 1}=-q(q-1)(q-2)\left(2 q^{7}-30 q^{6}+195 q^{5}-716 q^{4}+1613 q^{3}-2239 q^{2}+1775 q-617\right) \\
a_{33344(4), c, 2}=q(q-1)(q-3)\left(q^{2}-3 q+3\right)\left(q^{3}-7 q^{2}+16 q-11\right)(q-2)^{4}
\end{gathered}
$$




$$
\begin{gathered}
b_{33344(4), c, 1}=-q^{4}+10 q^{3}-43 q^{2}+93 q-82, \\
b_{33344(4), c, 2}=(q-3)\left(2 q^{5}-22 q^{4}+100 q^{3}-235 q^{2}+284 q-139\right), \\
b_{33344(4), c, 3}=-(q-3)\left(q^{3}-7 q^{2}+16 q-11\right)(q-2)^{4} .
\end{gathered}
$$

Three of the four eigenvalues of the $M D$ matrix are the inverses of the roots of $1+$ $b_{33344(4), c, 1} x+b_{33344(4), c, 2} x^{2}+b_{33344(4), c, 3} x^{3}$. These three eigenvalues, denoted $\lambda_{1}, \lambda_{2}$ and $\lambda_{3}$, contribute to the generating functions for all subgraphs that we consider on the two ends of the strip. The other eigenvalue is $\lambda_{4}=1$, which contributes to the generating functions for some subgraphs. In Table IV we show for some illustrative subgraphs on the two ends of the strips, whether or not $\lambda_{4}$ contributes to the generating function and some features of the boundaries $\mathcal{B}$ for each case. 
TABLE IV. Illustrative subgraphs for a strip of the $\left(3^{3} \cdot 4^{2}\right)$ lattice of type $(33344 b)$ with periodic boundary conditions in the transverse direction and transverse cross sections forming squares. The second column indicates whether or not $\lambda_{4}$ contributes to the generating function. The third column shows some

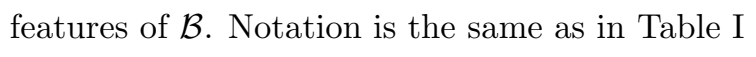

\begin{tabular}{|ccc|}
\hline \hline J,$I$ & does $\lambda_{4}$ enter? & features of $\mathcal{B}$ \\
\hline \hline $\mathbf{v}, \mathbf{v}$ & $\mathrm{N}$ & arcs \\
$\mathbf{v}, \mathbf{v}_{\mathbf{i}}, i=1, ., 6$ & $\mathrm{~N}$ & arcs \\
$\mathbf{v}_{\mathbf{1}}, \mathbf{v}_{\mathbf{i}}, i=1,2,3,4$ & $\mathrm{Y}$ & arcs and one enclosed region \\
$\mathbf{v}_{\mathbf{1}}, \mathbf{v}_{\mathbf{i}}, i=5,6$ & $\mathrm{~N}$ & arcs \\
$\mathbf{v}_{\mathbf{2}}, \mathbf{v}_{\mathbf{i}}, i=2,3,4$ & $\mathrm{Y}$ & arcs and one enclosed region \\
$\mathbf{v}_{\mathbf{2}}, \mathbf{v}_{\mathbf{i}}, i=5,6$ & $\mathrm{~N}$ & arcs \\
$\mathbf{v}_{\mathbf{3}}, \mathbf{v}_{\mathbf{i}}, i=3,4$ & $\mathrm{Y}$ & arcs and one enclosed region \\
$\mathbf{v}_{\mathbf{3}}, \mathbf{v}_{\mathbf{i}}, i=5,6$ & $\mathrm{~N}$ & arcs \\
$\mathbf{v}_{\mathbf{4}}, \mathbf{v}_{\mathbf{4}}$ & $\mathrm{Y}$ & arcs and one enclosed region \\
$\mathbf{v}_{\mathbf{4}}, \mathbf{v}_{\mathbf{i}}, i=5,6$ & $\mathrm{~N}$ & arcs \\
$\mathbf{v}_{\mathbf{5}}, \mathbf{v}_{\mathbf{i}}, i=5,6$ & $\mathrm{~N}$ & arcs \\
$\mathbf{v}_{\mathbf{6}}, \mathbf{v}_{\mathbf{6}}$ & $\mathrm{N}$ & arcs \\
\hline \hline
\end{tabular}



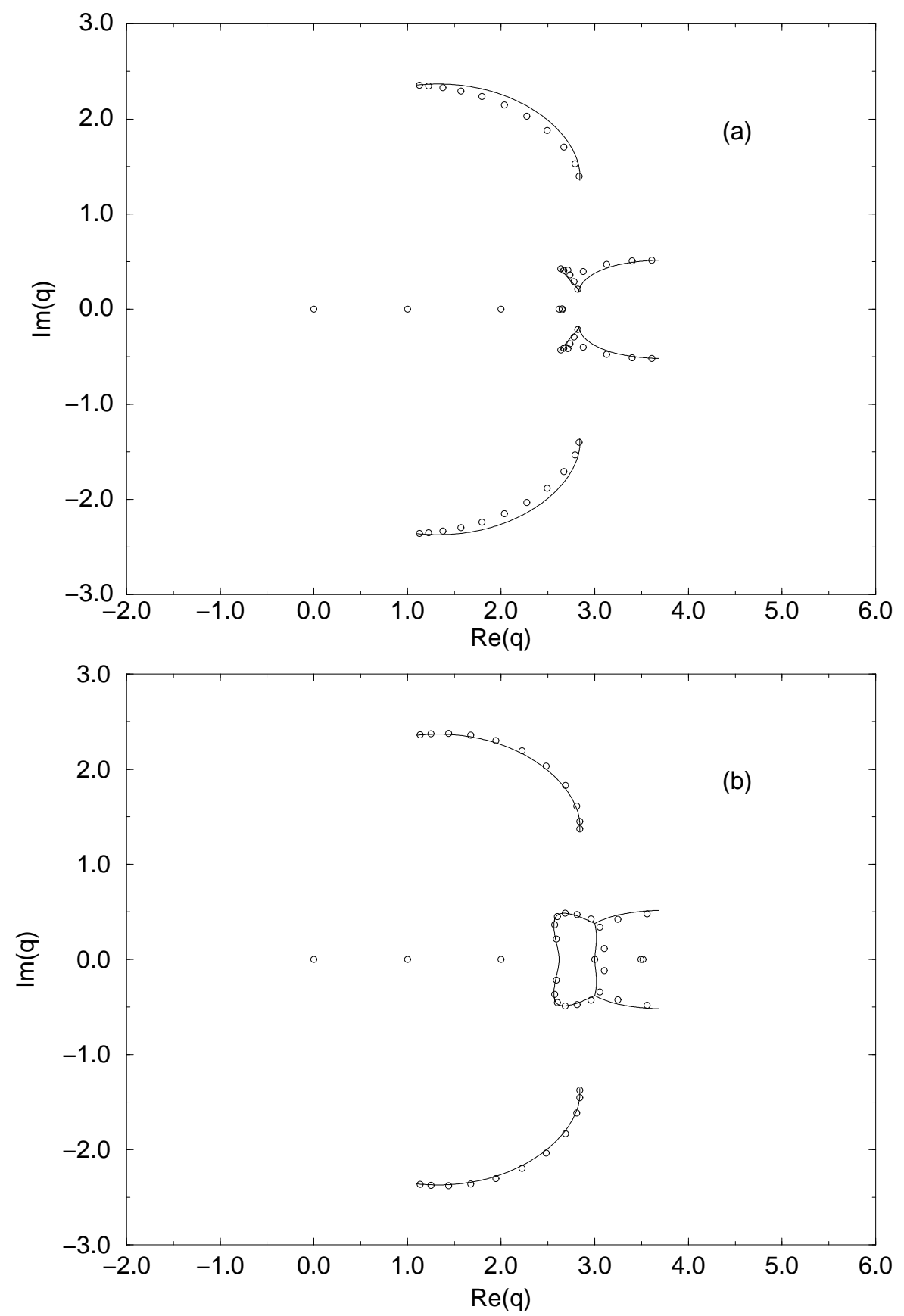
FIG. 8. Analytic structure of the function $W$ for the strip of the $\left(3^{3} \cdot 4^{2}\right)$ lattice of type $(33344 b)$ with periodic boundary conditions on the transverse direction, transverse cross sections forming squares, and length $L_{x}=\infty$. (a) corresponds to subgraphs on the free ends of the strip for which $\lambda_{4}$ does not enter in the generating function. (b) corresponds to cases where $\lambda_{4}$ enters in the generating function. For comparison, the zeros of the chromatic polynomial for a strip with $m=10$ repeating units ( $n=48$ vertices) and subgraphs described by (a) $\mathbf{v}\left[(b) \mathbf{v}_{\mathbf{1}}\right]$ on the right and left ends of the strip, are shown.

For subgraphs where $\lambda_{4}$ is not present in the generating function the analytic structure of the $W$ function, shown in Fig. 8(a), is formed by two pairs of complex-conjugate arcs which do not cross the real $q$ axis and do not enclose regions. There is a pair of multiple points at $q \simeq 2.83 \pm 0.19 i$, which can be seen more clearly in Fig. $\mathbb{U}(\mathrm{b})$ where the region in the complex $q$ plane around one of the multiple points is enlarged. For subgraphs where $\lambda_{4}$ is present in the generating function the analytic structure of the $W$ function, shown in Fig. 8(b), has arcs and a closed self-conjugate region, where $\lambda_{4}$ is leading. In the cases where $\lambda_{4}$ is not present in the generating function, the points $q \simeq 2.6243 \pm 0.4328 i$ correspond to arc endpoints, whereas in the cases for which $\lambda_{4}$ contributes to the generating function, these points lie within the enclosed region where $\lambda_{4}$ is leading and do not correspond to arc endpoints. The boundary $\mathcal{B}$ crosses the real $q$ axis at $q=\frac{3+\sqrt{5}}{2}=2.618 \ldots$ and at $q_{c}=3$. It is interesting to note that for a strip of the $\left(3^{3} \cdot 4^{2}\right)$ lattice of type $(33344 b)$ with periodic boundary conditions on the transverse direction and transverse cross sections forming squares, in all cases where $\mathcal{B}$ crosses the real $q$ axis, we have $q_{c}=3$, which coincides with $q_{c}$ for strips $G_{33344 b(4)}^{\prime}$ with free boundary conditions on the longitudinal and transverse directions. For both periodic and free boundary conditions on the transverse direction, the boundary $\mathcal{B}$ only cross the real $q$ axis for certain subgraphs on the longitudinal ends of the strip. 


\section{DISCUSSION AND CONCLUSIONS}

In Ref. [2] we have presented exact calculations of chromatic polynomials $P$ and asymptotic limiting $W$ functions for strip graphs of the form $J\left(\prod_{\ell=1}^{\infty} H\right) I$, where $J$ and $I$ are various subgraphs on the left and right ends of the strip, and $\left(\prod_{\ell=1}^{m} H\right)$ are strips of various regular lattices consisting of $m$-fold repetitions of subgraph units $H$. We have also studied the effects of using periodic as well as free boundary conditions in the transverse direction. For the respective strip graphs we have determined the loci $\mathcal{B}$ where $W$ is nonanalytic in the complex $q$ plane. We find that for some $J\left(\prod_{\ell=1}^{\infty} H\right) I$ strip graphs, $\mathcal{B}$ (i) does depend on $I$ and $J$, and (ii) can enclose regions in the $q$ plane. In the cases with free boundary conditions in the longitudinal and transverse directions that were studied in Ref. [2] the boundary $\mathcal{B}$ enclosed regions when the end subgraphs $J$ and $I$ were non-planar graphs. In the present paper we present an example for this class of strip graphs in which $\mathcal{B}$ encloses regions even for planar end graphs. The bulk of the specific strip graph that exhibits this property is a part of the $\left(3^{3} \cdot 4^{2}\right)$ Archimedean lattice. The boundary $\mathcal{B}$ for these strip graphs have multiple points and cusps. We have also studied the effects of taking different types of strips of the same uniform lattice as well as of using periodic and free boundary conditions in the transverse direction. It is interesting to note that in all cases where $\mathcal{B}$ crosses the real $q$ axis, the maximum crossing points are the same, namely $q_{c}=\chi=3$.

\section{Acknowledgments}

Discussions with Professors Martin Roček and Robert Shrock are gratefully acknowledged. I would also like to thank Professor Robert Shrock for helpful comments on the manuscript. This research was supported in part by the NSF grant PHY-97-22101. 
[1] M. Roček, R. Shrock, and S.-H. Tsai, Physica A252 (1998) 505.

[2] M. Roček, R. Shrock, and S.-H. Tsai, Stony Brook preprint ITP-SB-98-29, Physica A, in press.

[3] R. B. Potts, Proc. Camb. Phil. Soc. 48 (1952) 106.

[4] F. Y. Wu, Rev. Mod. Phys. 54 (1982) 235; errata, ibid. 55 (1983) 315.

[5] R. C. Read, J. Combin. Theory 4 (1968) 52; R. C. Read and W. T. Tutte, "Chromatic Polynomials", in Selected Topics in Graph Theory, 3, eds. L. W. Beineke and R. J. Wilson (Academic Press, New York, 1988; R. Nelson and R. J. Wilson, eds., Graph Colourings (Longman, Essex, 1990).

[6] R. Shrock and S.-H. Tsai, ITP-SB-98-19, Physica A, in press.

[7] B. Grünbaum and G. Shephard, Tilings and Patterns (Freeman, New York, 1987).

[8] R. Shrock and S.-H. Tsai Phys. Rev. E56 (1997) 4111.

[9] R. Shrock and S.-H. Tsai, J. Phys. A 30 (1997) 495.

[10] R. Shrock and S.-H. Tsai, Phys. Rev. E55 (1997) 5165.

[11] R. Shrock and S.-H. Tsai, Phys. Rev. E55 (1997) 6791; E56 (1997) 2733; S.-H. Tsai, Phys. Rev. E57 (1998) 2686.

[12] S.-H. Tsai, Ph.D. thesis, SUNY Stony Brook, April, 1998.

[13] S. Lefschetz, Algebraic Geometry (Princeton Univ. Press, Princeton, 1953); R. Hartshorne, Algebraic Geometry (Springer, New York, 1977).

[14] R. J. Baxter, J. Phys. A 20 (1987) 5241. 